\title{
Strict conditionals: a negative result *
}

\author{
Jan Heylen Leon Horsten
}

\begin{abstract}
Jonathan Lowe has argued that a particular variation on C.I. Lewis' notion of strict implication avoids the paradoxes of strict implication. In this paper it is argued that Lowe's notion of implication does not achieve this aim. Moreover, a general argument is given to the effect that no other variation on Lewis' notion of constantly strict implication describes the logical behavior of natural language conditionals in a satisfactory way.
\end{abstract}

\section{Indicative conditionals and strict implication}

In reaction to Russell's interpretation of conditional sentences as classical material implications, C.I. Lewis suggested that it would be more appropriate to interpret natural language conditionals as strict implications. He claimed that the truth-conditions of a sentence of the form 'if $p$ then $q$ ' are given by the sentence 'Necessarily, not $p$ or $q$ ' Lewis (1912).

Russell and Lewis were speaking at cross purposes. Russell was mainly interested in the logical meaning of conditional expressions in the restricted context of mathematical proofs, whereas C.I. Lewis wanted to express the logical meaning of indicative conditionals in natural language in general. And it is true that in mathematical proofs, the meaning of conditional assertions can be taken to be expressed by the corresponding material implications. But the so-called paradoxes of material implication to which C.I. Lewis drew Russell's attention do show that material implications do not capture the truth-conditions of conditional expressions as they are generally used in daily speech. Attempts have been made to relegate the paradoxes of material implication to pragmatics, by classifying the paradoxes of material implication as true assertions which violate some of the Gricean conversational implicatures. But the attempts that are made so far are generally regarded as unsatisfactory. ${ }^{1}$

In the past decades, some philosophers have sought to cast doubt on the assumption that natural language conditionals have truth values at all. On this view, indicative conditionals can merely be considered more or less acceptable or assertible, but never true or false. ${ }^{2}$ These philosophers may well be right, but in this paper we will assume, along with C.I. Lewis and Russell and a score of contemporary philosophers, that conditionals do have truth-conditions. We shall also assume that all indicative conditionals share a common logical form, even though we recognize that this too is an assumption that could be challenged.

\footnotetext{
${ }^{*}$ Research for this paper was supported by grant G.0239.02 of the Fund for Scientific research -Flanders, which is gratefully acknowledged.

${ }^{1}$ Even the neo-griceans admit this. See, for instance, Levinson (2000, p. 208-209).

${ }^{2}$ See, for instance, Adams (1998), Edgington (1995).
} 
Lewis' idea of interpreting conditionals as strict implications becomes a determinate proposal against the background of a set of laws governing the notion of necessity a definite interpretation of the concept of necessity involved. About the notion of necessity employed Lewis had logical necessity in mind. With respect to the laws of necessity, Lewis himself proposed several alternatives, some of which have since then become 'standard' systems of (propositional) modal logic.

It was pointed out early (by Quine, for instance) that Lewis' theory suffers from a confusion between genuine conditional statements and metalinguistic statements. ${ }^{3}$ Sometimes Lewis discusses if / then-statements, but then he lapses into talk about statements expressing (logical) implication and consequence. So let be explicit here, and state that the theory intends to uncover the logical form of natural language statements of the form 'if $p$ then $q$ '. Also, we restrict ourselves here to indicative conditionals. Specifically, we remain neutral about the question whether counterfactual conditionals have the same truth-conditionals as indicative conditionals. Also, we leave out two classes of if / then-statements which do not express genuine conditionals. These are the so-called Biscuit Conditionals and the Dutchman Conditionals. Biscuit Conditionals are 'conditional' statements of which the truth-conditions coincide with the truth-conditions of their consequent. An example is:

If you want a glass of wine, there is an open bottle in the refrigerator.

Dutchman Conditionals are "conditional" statements of which the truth-conditionals are equivalent to the truth-conditions of their antecedents. An example is:

If that's a snake then I am a Dutchman.

So both Biscuit Conditionals and Dutchman Conditionals are conditional in name only; they are really categorical assertions of the consequent and of the antecedent, respectively.

Since Lewis' days, it has become clear that if natural language conditionals are interpreted as strict conditionals, certain odd-sounding judgements concerning the validity of sentences and inferences involving conditional expressions remain. These have become known as the paradoxes of strict implication. For instance, conditionals of the form 'If $0=1$, then the sun shines tomorrow' are generally viewed as un-assertible, even though the corresponding strict implication is valid in every standard system of modal logic. Philosophers agree that the truth-conditions of a strict implication are weaker than the truth-conditions of a natural language conditional.

\section{Variations}

In view of the paradoxes of strict implication, most philosophers have abandonded C.I. Lewis' idea of interpreting ordinary language conditionals as strict implications. But E.J. Lowe has rightly observed that this was a hasty conclusion Lowe (1995, p. 48). He tried to amending Lewis' proposal in such a way that the paradoxes of strict implication disappear.

Lowe's proposal is that conditionals of the form 'If $p$ then $q$ ' ought to be interpreted as follows Lowe (1995, p. 49):

$$
\square(\neg p \vee q) \wedge(\diamond p \vee \square q)
$$

\footnotetext{
${ }^{3}$ For a detailed historical account of this matter, see Neale (2000, Part II).
} 
Thus a variation on Lewis' proposal is generated. This proposal has the virtue of making the conditional 'If $p$ and not $p$, then the sun shines tomorrow' invalid. True, one could achieve this effect in a simpler way, namely by reading the conditional just as $\square(\neg p \vee q) \wedge \diamond p$. But this would make conditionals such as 'If $0=1$ and $1=1$, then $1=1$ ' come out false, even though they seem perfectly true Lowe (1995, p. 48). ${ }^{4}$ In other words, the simpler proposal would generate new paradoxes of strict implication; the reading of conditionals proposed by the simple example would be too strong. Lowe's more subtle reading of conditionals, however, makes 'If $0=1$ and $1=1$, then $1=1$ ' come out true, which is perfectly in accordance with our intuitions.

Nevertheless Lowe's variation on Lewis' idea also generates new 'paradoxical' inferences. The reason is that in the context of mathematical proofs, Lowe's proposal is at variance with the judgements generated by Russell's proposal. Consider the sentence 'If $2=3$, then $2+1=3+1$ '. On the face of it, this looks like a perfectly correct conditional statement. But on Lowe's theory, it can never be correctly asserted. In this way the truth-conditions of Lowe's strict conditional appear more restricted than that of indicative natural language conditionals. Note that nothing hinges on our counterexample being a sentence of mathematics. For instance, the sentence

If I am my father, then my father is my father's father

would do equally well. But the message of Lowe's proposal is that this is not the end of the matter. For a lover of strict conditionals can try other variations on Lewis' idea. As an analysis of sentences of the form 'If $p$ then $q$ ' he (she) can in principle propose any reading

$$
\square(\neg p \vee q) \wedge X,
$$

where $X$ is a condition in $p, q, \square$, and the connectives of classical propositional logic. ${ }^{5}$

\section{The argument}

We adopt the abstract viewpoint and develop an argument to show that Lowe-like variations on Lewis' proposal can never capture the truth-conditions of natural language indicative conditionals. It will be argued that variations on Lewis' strict implication are either too weak in some respects, or too strong. That is, the reading that they provide either classifies certain intuitively unacceptable conditional statements as true, or it qualities certain intuitively acceptable conditional statements as false.

To this end, we consider the lattice of propositions that can be expressed in terms of $p, q, \square$, and the classical propositional connectives. This lattice can be partially ordered according to information content, with $\perp$ (Falsum) at the top and $T$ (True) at the bottom.

The size of this lattice is of the order $2^{32}$ Carnap (1946, p. 48), which at first sight seems a bit discouraging. But we do not have to survey all these propositions. We already know that the reading $\square(\neg p \vee q)$ is too weak. We will consider the propositions which lie just above $\square(\neg p \vee q)$ in the lattice. We will argue for all of them that as

\footnotetext{
${ }^{4}$ The example Lowe gives is: 'If $n$ were the greatest natural number, then there would be a natural number greater than $n$.' But this example is perhaps less clear, for it appears that here $n$ plays the role of a free numerical variable.

${ }^{5}$ It is perhaps also possible to regard the extra condition $X$ as the associated conversational generalized conversational implicature of the indicative conditional.
} 


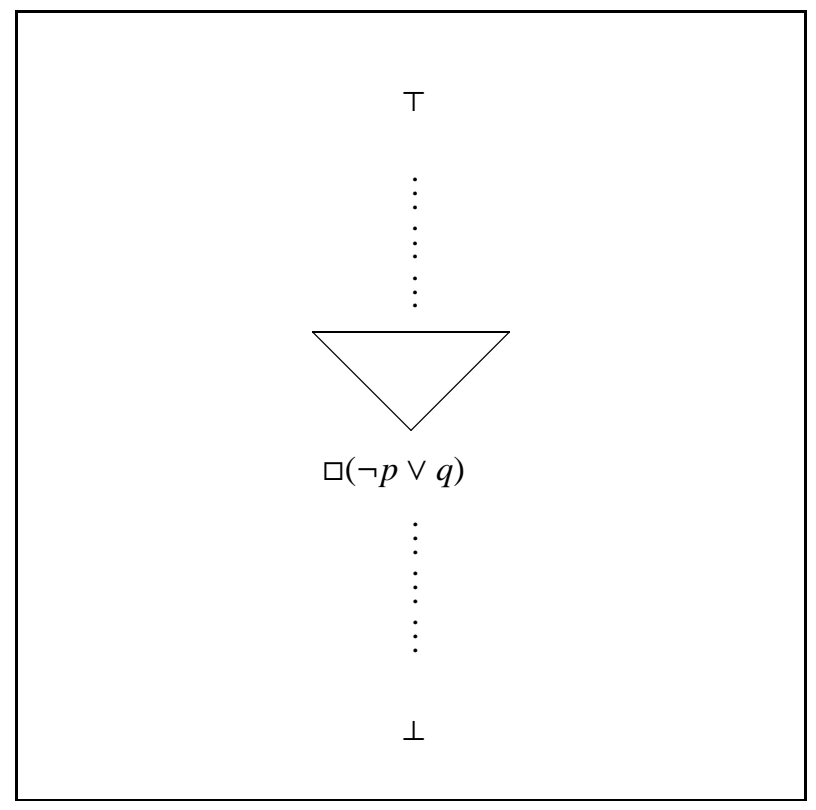

Figure 1: Lattice

readings of indicative natural language conditionals, they are either too weak or too strong.

Our main argument makes two assumptions. The second of these assumptions is only temporary: it will be removed later in the paper.

The first assumption is that the correctness of a reading of conditional statements does not hinge on laws governing iterations of modal operators. If the notion of necessity involved is governed by the $\mathbf{S 5}$ laws, then iterations of modalities can always be eliminated. But even if the laws governing necessity are significantly weaker, it would be scarcely imaginable that the correct interpretation of conditionals essentially involves nested modalities. The resulting readings would be just too complicated for humans to effectively use in ordinary reasoning. So we shall assume that the putative explications of indicative conditionals do not contain nested modal operators. Equivalently, we proceed on the assumption that only the $\mathbf{S 5}$ lattice is relevant for our investigation.

The second assumption is that $X$ can be taken to be a purely modal condition. In other words, all occurrences of the propositional atoms $p, q$ in $X$ are in the scope of $\square$. For counterfactual conditionals such an assumption would be contentious. For some claim that the correctness of a counterfactual conditional entails the factual falsity of its antecedent, and this factual falsity is usually regarded as not being entailed by the modal relation in which $p$ and $q$ stand. But, as we stated at the outset, in this paper we make no claims concerning the logical form of counterfactuals. Concerning indicative conditionals, it seems at least prima facie less likely that their logical meaning contains an irreducibly factual component. At any rate, this assumption will ultimately prove to be inessential to our argumentation.

For the rest, our argument makes use of only very weak modal assumptions. Specifically, the basic modal system $\mathbf{T}$ suffices for our argument, to which we now turn. 
The proposition that is expressed by $X$ can be put in disjunctive normal form. In general, $\diamond(\neg p \vee q)$ should not be one of the disjuncts, for then the resulting $X$ is implied by $\square(\neg p \vee q)$. So we turn to the slightly weaker

$$
\diamond(p \vee q) \vee \diamond(p \vee \neg q) \vee \diamond(\neg p \vee \neg q)
$$

This value for $X$ is equivalent to $\neg \square \perp$. Therefore conjoining it does not strengthen the content of $\square(\neg p \vee q)$. The same holds for disjunctions of two of the disjuncts of the preceding formula, such as $\diamond(p \vee q) \vee \diamond(p \vee \neg q)$. They too do not increase the strength of $\square(\neg p \vee q)$.

So we incrementally increase the strength, and look at the following putative values for $X$ :

$$
\begin{array}{r}
\diamond p \vee \diamond q \\
\diamond p \vee \diamond \neg q \\
\diamond \neg p \vee \diamond \neg q
\end{array}
$$

It can be shown that each of these result in a reading of conditionals that is too strong. We first consider the reading $\square(\neg p \vee q) \wedge(1)$. Consider the sentence 'If $2=3$ then $2+1=3+1$ '. This sentence is intuitively true. But if it is interpreted in accordance with $\square(\neg p \vee q) \wedge(1)$, it is judged false. The following theorem of number theory offers a less childlish sort of example, consider the following theorem of number theory:

If Goldbach's conjecture is true, then every number greater than 17 is the sum of three distinct primes.

It was proved that this conditional statement can be strengthened to an equivalence. Now suppose that contrary to expectation Goldbach's conjecture turns out to be false. Then the consequence of the conditional statement under consideration would also be false, and our reading $\square(\neg p \vee q) \wedge(1)$ would judge the conditional incorrect. But surely the conditional statement would still be true. Granted, once is is known that Goldbach's conjecture is false, it will be more appropriate to assert the conditional connection in a counterfactual manner. But that is no more than an application of the Gricean maxim of informativeness. The corresponding counterfactual would convey that the antecedent is known to be false. The indicative conditional would still be correct - we would not have to rewrite the mathematics textbooks. But it would be pragmatically defective: our assertion would not be maximally informative.

Next, we consider the reading $\square(\neg p \vee q) \wedge(2)$. Here too we find a counterexample: 'If $0=1$ and $1=1$, then $1=1$ '. Again this sentence seems intuitively true if it has any truth-value at all. Yet on the reading under consideration, it comes out false. Lest this example is also considered a bit on the simplistic side, consider the following assertion:

If Frege Arithmetic is consistent, then Peano Arithmetic is consistent.

Russell taught us that Frege Arithmetic is inconsistent. The antecedent of this conditional assertion therefore is necessarily false, whereas the consequent is necessarily true.

Finally, we look at $\square(\neg p \vee q) \wedge(3)$. This proposal can be countered with the sentence 'If $2=2$ then $2+1=2+1$ '. After all, it is a theorem of arithmetic. But the proposed reading makes it false. So we conclude that all these readings are too strong. 
As before, nothing hinges on the counterexamples being arithmetical statements. Nonmathematical statements serving the same purpose are readily found, as the reader can check for him- or herself.

We also have to consider the following putative values for $X$ :

$$
\begin{aligned}
& \square \neg p \vee \diamond q \\
& \diamond \neg p \vee \square q
\end{aligned}
$$

To both of them, the sentence

If I am my father, then Belgium is a dictatorship

serves as a counterexample. Intuitively, this conditional does not ring true. But according to the two readings under consideration, they are true. So the readings generated by these two putative values of $X$ are too weak. So again we incrementally move up the lattice, and encounter as a putative value of $X$ the formula

$$
\square \neg p \vee \square q .
$$

But this value yields a logical interpretation of conditional statements that is too strong. This is witnessed by the following statement:

If I have 3 Euros in my pocket, then I have more than 2 Euros on me.

This seems a true conditional statement. But both its antecedent and its consequent express contingent propositions. So the logical reading under consideration makes it false.

Modal side conditions $X$ in only one variable ( $p$ or $q$ ) need not be considered, for they are all stronger than the minimal side conditions that we have considered and found too strong. For similar reasons, side conditions in which complex propositional formulas occur in the scope of a modal operator need not be considered. They either are entailed by $\square(\neg p \vee q)$ and therefore carry no extra information, or entail a modal side condition in which only proposition letters or negations thereof occur in the scope of a modal operator and which was found to be too strong. As an example, consider the side condition $\square[(p \wedge q) \vee(\neg p \wedge \neg q)]$. This condition is stronger than $\diamond \neg p \vee \diamond q$ yet weaker than $\square \neg p \vee \square q$. So ought we not consider it? No, for it is stronger than $\diamond \neg p \vee \diamond \neg q$, which was shown to be already too strong.

Summarizing, the situation can be described thus. Just above $\square(\neg p \vee q)$ in the lattice, we find either readings which are too strong ((1), (2), (3)) or which are still too weak $((4),(5))$. But just above these readings which are still too weak, we find one single reading $((6))$ which is again too strong. So we have "capped" the reading $\square(\neg p \vee q)$ by readings that are either too weak or too strong.

Now we can see that the second assumption of our argument is not essential. We shall reconsider the readings that are too weak, and show that conjoining even the weakest extra factual condition to it results in a reading that is too strong.

First, consider Lewis' basic reading $\square(\neg p \vee q)$. The weakest factual conditions that can be conjunctively added to this reading are $p \vee q, p \vee \neg q, \neg p \vee q, \neg p \vee \neg q$. Conjoining $p \vee \neg q$ yields a reading which is too strong. For a counterexample,

If we are brains in a vat, then the outside world exists. 


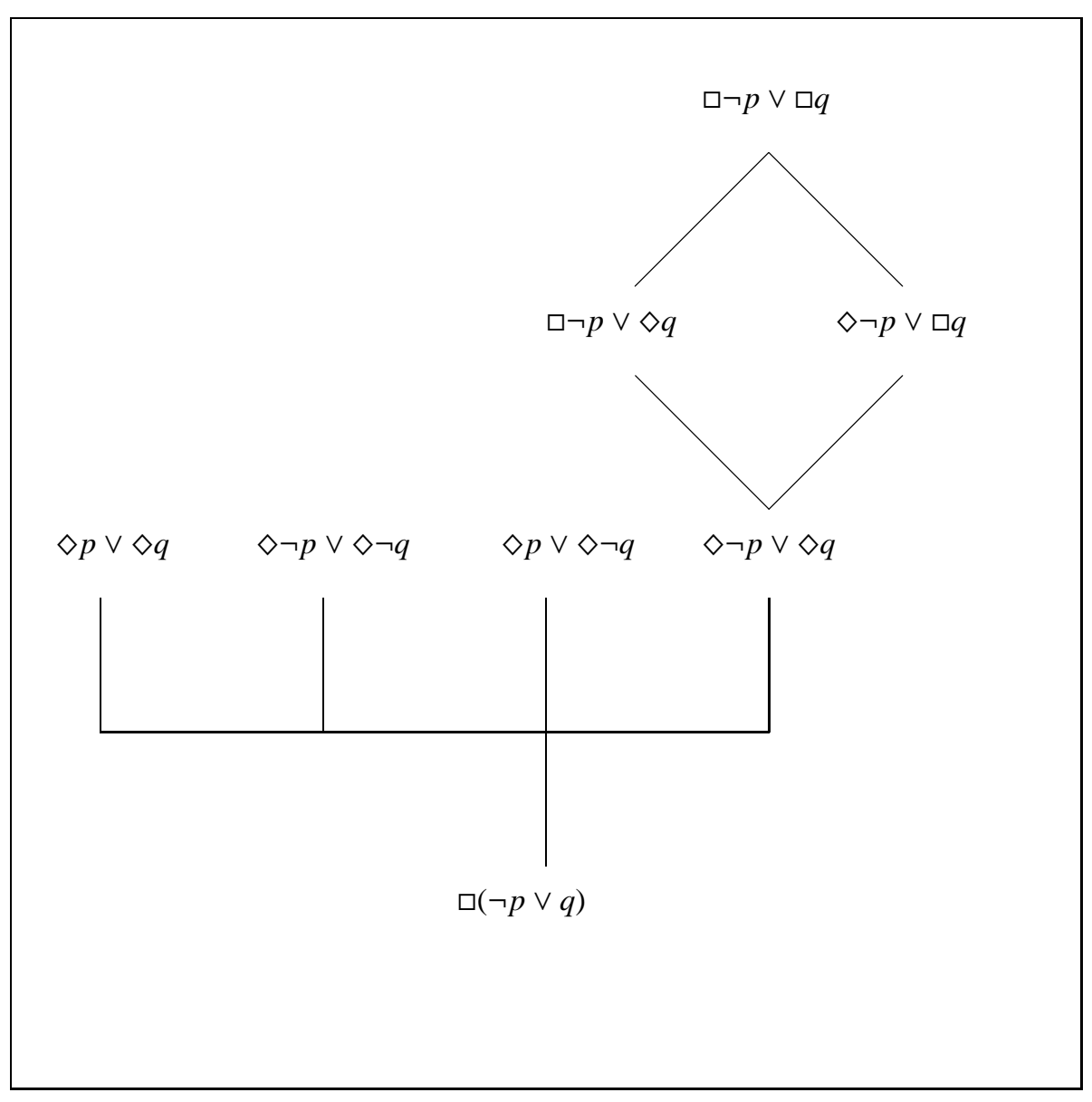

Figure 2: Capping 
seems to be a correct assertion, for if we are brains in a vat, then at least the vat must exist. Yet it seems eminently plausible that the antecedent is false and the consequent is true. So the conditional statement does not satisfy the side condition, and therefore it is wrongly classified as false by our reading.

We now consider $p \vee q$ and $\neg p \vee \neg q$. For $p \vee q$, consider the assertion

If there are seven planets in our solar system, then the number of planets is prime.

as uttered by someone in the eighteenth century, after the discovery of Uranus, but before the discovery in the nineteenth century of Neptune. And for $\neg p \vee \neg q$, consider the assertion

If the earth revolves around the sun, then the nearer of the fixed stars should appear to move relative to the farther ones.

as uttered by a sixteenth century astronomer. These readings are judged incorrect by the respective readings. Yet they appear perfectly sound conditional assertions. This leaves us with $\neg p \vee q$. Conjoining $\neg p \vee q$ adds no information. (This is one of the places in our argument where the T-axiom is used.) For it is this principle that implies that the logical content of $\neg p \vee q$ is weaker than that of $\square(\neg p \vee q)$. So we contemplate strengthening by conjoining a slightly stronger factual condition: $\neg p$, or $q$. But each of these is stronger than conditions which have already been shown to yield readings that are too strong. Now we have surveyed all factual conditions just above $\square(\neg p \vee q)$ (see Figure 3).

Secondly, consider the readings (4) and (5). The weakest factual conditions that can be conjunctively added to these readings again are $p \vee q, p \vee \neg q, \neg p \vee q, \neg p \vee \neg q . p \vee \neg q$ yields a reading that is too strong. The counterexample about Frege Arithmetic that was described earlier again illustrates this. As for $p \vee q$ and $\neg p \vee \neg q$, we first contemplate adding $p \vee q$ as an extra conjunct of $X$. The resulting modifications (4a) and (5a) of (4) and (5) are too strong. To these, we formulate the following counterexample:

If I am my father, then my father's first name is the same as mine.

This is an acceptable conditional with an impossible antecedent and a contingently false consequent. Yet according to (4a), this conditional is false. (5a) too would classify this conditional statement as false. So readings (4a) and (5a) are too strong. Adding $\neg p \vee \neg q$ as extra conjunct of $X$, results in readings (4b) and (5b). A counterexample to this is the statement 'If $3=3$, then $3+1=3+1$ '. Again this is a correct conditional statement, but it is classified by (4b) and (5b) as false. So these readings are also too strong. Now all we are left with is $\neg p \vee q$, which is too weak. So we must again contemplate adding something slightly stronger to (4) and (5), namely $\neg p$ or $q$. But these conditions are stronger than the conditions $\neg p \vee \neg q$, and $p \vee q$, respectively, which have been shown to yield readings that are too strong. Thus the argument is concluded.

We now see that it is no accident that Lowe's proposed variation Lewis' idea does not work. The extra condition $X$ which lifts the reading $\square(\neg p \vee q)$ to an interpretation of conditionals which is exactly strong enough, cannot be expressed in terms of $p, q$, $\square$, and the connectives of classical propositional logic.

\section{Classes of indicative conditionals}

The outcome of the argument is on the whole not unexpected. Most philosophers of language today would regard it as unlikely that a variation on C.I. Lewis' strict implica- 
tion can accurately describe the logical behaviour of indicative conditional statements. This is witnessed by the fact that many of the contemporary philosophical theories about indicative conditionals fall outside the scope of our negative result. Nevertheless, the results of this paper do affect some recent theories of indicative conditionals, such as that of Lowe but also that of Warmbrōd. ${ }^{6}$ The upshot of this paper is that most philosophers of language and philosophical logicians rightly believe that to arrive at the correct logical interpretation of indicative conditionals, a new idea is needed. And this involves challenging either to challenge some of our intuitive judgments concerning the truth-values of our examples or one or more of the presuppositions of our negative result.

A first option is, as noted earlier, to challenge some of the intuitive judgments concerning conditionals that were adduced to refute proposed logical interpretations of indicative conditionals. Lowe himself, for instance, questions whether 'If $2=3$, then $2+1=3+1$ ' has a truth-value. He believes that this sentence is assertible as long as it is simply regarded as an instantiation of the true universal generalization 'For all natural numbers $m$ and $n$, if $m=n$ then $m+1=n+1$ '; but taken as an assertion specifically about the numbers 2 and 3, it is highly paradoxical and has no intuitive truth value. For if $2=3$, then arithmetic as we know it is a complete mistake, so the laws of addition cannot be trusted. But this seems hard to maintain. For it amounts to denying the validity of the rule of universal instantiation which is valid even in partial logic. It must be admitted that many philosophers of language today deny that conditional statements with an impossible antecedent can ever have a truth-value. For some such conditionals this may have intuitive appeal ('If $0=1$, then it will rain tomorrow'). But for a sentence such as 'If $2=3$, then $2+1=3+1$ ', the immediate intuitive appeal of claiming that it has no truth value seems limited. And its appeal seems to diminish further if the example is replaced by a less elementary one, such as the example of Goldbach's conjecture that was discussed earlier.

A second option that our argument does leave open is to explicate the logical behaviour of conditionals not just in terms of the propositional variables, the usual logical connectives of propositional logic and the modal operators $\square$ and $\diamond$. One can, for instance, introduce a comparative possibility operator $i$ ('It is more possible that ... than that ...'). This is exactly what David Lewis has done when he introduced the notion of a variably strict conditional as the logical form of counterfactual conditionals. With the help of a comparative possibility operator one can define a counterfactual operator $\square \rightarrow$ as follows:

$$
p \square \rightarrow q=_{d f} \neg \diamond p \vee((p \wedge q)<(p \wedge \neg q))
$$

So the idea is that if $p$ were the case, $q$ would have been the case is (non-vacuously) true if and only if it is more possible that $p$ and $q$ are both true than that $p$ is true but $q$ is not. Lewis himself applied the notion only to counterfactual conditionals, but some authors, such as Gillies, have argued that it may also be applied to indicative conditionals. At any rate, variably strict conditionals for the most part fall outside the scope of our argument, because variably strict conditionals cannot be defined in terms of the propositional variables, the usual logical connectives of propositional logic and the common modal operators.

A counterfactual conditional is vacuously true whenever it has an impossible antecedent. David Lewis was well aware that paradoxes emerge at this point. He notes

\footnotetext{
${ }^{6}$ Warmbrōd (1983) and of von Fintel (1999)
} 
that some counterfactual conditionals with impossible antecedents are unassertible. For example:

If there were a largest prime $\mathrm{p}$, pigs would have wings.

Lewis himself doubted whether such conditionals could be deemed false, but according to the view adopted in this paper these conditionals should be assigned a truth value (i.c. 'false'). It occurred to Lewis that one possible reply would be to install a condition that would ensure that the antecedent of a counterfactual conditional should always be possible. His proposal was to introduce a new counterfactual operator with the following contextual definition:

$p \square \Leftrightarrow q=_{d f}(p \wedge q)<(p \wedge \neg q)$

It is easily derivable that p should be possible whenever $p \square \Leftrightarrow q$ is true. But Lewis also pointed out that certain counterfactual conditionals with impossible antecedents seem to be true. For example:

If there were a decision procedure for logic, there would be one for the halting problem.

A question analogous to the question we considered in this paper arises, namely the question whether there is any variation on the notion of a variably strict conditional such that all paradoxes can be avoided.

At this point one may wonder whether it is possible to extend our argument so as to cover the variably strict conditionals also. After all, constantly strict conditionals are just a special kind of variably strict conditionals. Indeed, whenever a constantly strict conditional is true, the corresponding variably strict conditional is true. Moreover, we have just seen that the notion of variably strict conditionals also gives rise to paradoxes, and that at least one variation on that notion has been proposed to escape from the paradoxes. It would not be an easy task, however, to extend our argument to variably strict conditionals. Briefly and roughly put, the difficulty consists in the fact that one can abstract many kinds of possibilities from the comparative possibility ordering. For instance, logical tautology corresponds to maximal possibility and logical contradiction corresponds to minimal possibility, whereas physical possibility will be more-thanminimal but also less-than-maximal possibility. This raises the problem that one should have to consider many lattices, one for each kind of possibility abstracted from the comparative possibility ordering. Our capping procedure is not suited for this task. One should nevertheless not conclude that our intuitions regarding the paradoxes are necessarily better served by a logic of variably strict conditionals.

Thirdly, some maintain that the logical interpretation of indicative conditionals is indeed given by a necessary implication reading, but hold that contextual factors influence the interpretation of the notion of necessity involved. ${ }^{7}$ To put it somewhat schematically, one might say that the idea is that natural language conditionals are, on the semantic level constantly strict conditionals whereas they are, on the pragmatic level, variably strict conditionals.

As long as only possible worlds are involved in the interpretation of the notion of necessity, this will be of no help in avoiding the conclusion of the preceding argument. The laws of modal logic that are appealed to still go through. For if only possible worlds are involved, even when this set of possible worlds is restricted, sentences that

\footnotetext{
${ }^{7}$ This idea is clearly described in von Fintel (1999, p. 130). Lowe (1983, p. 357) asserts that this phenomenon applies to indicative conditionals, and Lowe (1995, p. 55) states that this is the case for counterfactuals. It is also a key component of the theory of Warmbrōd.
} 
are necessarily true on the context-insensitive reading will still be necessarily true on the contextual reading, and sentences that were impossible on the context-insensitive reading remain impossible after contextual relativisation. Of course in some contexts, contingently true (false) modal sentences become false (true) by contextual restriction of the set of possible worlds. But as long as for each of the examples that were adduced, at least one context can be construed for which the evaluation given of that example is correct, the argument goes through. And we maintain that is the case for the examples that we have given. That Lowes theory only involves possible worlds can be gathered from the fact that his logic of necessity is closed under the rule of necessitation. ${ }^{8}$ The same is true for Warmbrōd's theory. Even though he does not provide an axiomatisation of the modal logic behind his proposal, he asserts that it must be closed under necessitation. ${ }^{9}$

A more thoroughgoing contextualist theory might involve impossible worlds. These might be invoked in order represent contextually determined situations in which impossible states of affairs such as the falsehood of certain logical or mathematical facts are true. According to such theories, the modal operator will not in each context be governed by an extension of the normal modal logic T. On such readings of the modal operator, the truth-value of some of the examples adduced in the previous section in the light of certain variations of C.I. Lewis strict implication reading may indeed change. This would mean that the argument no longer goes through: we have arrived at a pragmatic escape from the conclusion of the argument. ${ }^{10}$

Fourthly, one could in a Gricean style view as conversational implicatures the extra conditions $X$ that were conjoined to the strict implication reading of indicative conditionals, and try to explain away some of our counterexamples by finding reasons why the expected implicatures do not apply. It is not clear to us at the moment how promising such an approach would be.

A fifth option would be to drop the assumption that a common logical form is shared by all indicative conditionals but maintain that a natural subclass of indicative conditionals is governed by a strict implication. This might involve isolating a subclass of indicative conditional assertions as expressing inferential conditionals, and arguing that the logical behaviour of these conditionals is accurately described by Lewis' strict implication or a variation on it. There may actually be something in this. For one has the feeling that the intuitive basis of our assent to conditionals such as 'If $2=3$, then $2+1=3+1$ ' is rooted in the existence of a derivational connection between $2=3$ and $2+1=3+1$. It seems that the conversational context can in some situations allow us to interpret an if/then-statement in an inferential way. Very roughly, the picture might be that if/then-statements can have at least three logical interpretations. When an if/thenstatement is used to express a law-like connection, its logical form is explicated by David Lewis' variably strict implication. But an if/then-statement can also be used to express a (subjective) conditional expectation of the speaker. In that case, Adams' interpretation in terms of conditional probability is appropriate. And to conclude, an if/then-statement can express an inferential relation. In this case, C.I. Lewis' constantly strict implication or a variation thereof supplies the correct logical interpretation. It exceeds the scope of this paper to work out this suggestion in detail.

These options are all left open by our argument. But we maintain on the grounds of the considerations that we have brought forward that if conditionals generally have

\footnotetext{
${ }^{8}$ Lowe (1983, p. 360)

${ }^{9}$ Warmbrōd (1983, p. 265, n. 21).

${ }^{10}$ Thanks to an anonymous referee for pointing this out.
} 
truth values, then the intuitive judgments concerning them will be along the lines set out by Lowe. And in that sense, the negative result of this paper seems to leave little room to maneuver for those who want to defend a variation on C.I. Lewis' proposal for all indicative conditionals.

\section{References}

Adams, E. 1998. A Primer of Probability Logic. CSLI Publications.

Carnap, Rudolf. 1946. Modalities and Quantification. Journal of Symbolic Logic, 11, 33-64.

Edgington, Dorothy. 1995. On Conditionals. Mind, 104, 235-329.

Levinson, Stephen C. 2000. Presumptive Meanings. The theory of generalized conversational implicatures. MIT Press.

Lewis, Clarence I. 1912. Implication and the Algebra of Logic. Mind, 21, 522-531.

Lowe, E. J. 1983. A simplification of the logic of conditionals. Notre Dame J. Formal Logic, 24(3), 357-366.

Lowe, E.J. 1995. The Truth about Conditionals. The Philosophical Quarterly, 45, 41-59.

Neale, Stephen. 2000. On a Milestone of Empiricism. In: Kotatko, P., \& Orenstein, A. (eds), Knowledge, Language, Logic: Questions for Quine. Kluwer Academic Publishers.

von Fintel, Kai. 1999. The Presupposition of Subjunctive Conditionals. Pages 2944 of: Sauerland, Uli, \& Percus, Orin (eds), The Interpretive Tract, vol. 25. MIT Working Papers in Linguistics.

Warmbrōd, Kenneth. 1983. Epistemic Conditionals. Pacific Philosophical Quarterly, 64, 249-265. 